\section{Óbitos neonatais precoces: análise de causas múltiplas de morte pelo método Grade of Membership}

\author{
Early neonatal mortality: an analysis of multiple \\ causes of death by the Grade of Membership \\ method
}

\author{
1 Secretaria Municipal de \\ Saúde de Belo Horizonte, \\ Belo Horizonte, Brasil. \\ 2 Faculdade de Medicina, \\ Universidade Federal de \\ Minas Gerais, \\ Belo Horizonte, Brasil. \\ ${ }^{3}$ Faculdade de Ciências \\ Econômicas, Universidade \\ Federal de Minas Gerais, \\ Belo Horizonte, Brasil. \\ Correspondência \\ E. França \\ Departamento de \\ Medicina Preventiva e Social, \\ Faculdade de Medicina, \\ Universidade Federal \\ de Minas Gerais. \\ Av. Alfredo Balena 190, \\ sala 10020, \\ Belo Horizonte, $M G$ \\ 30130-100, Brasil. \\ efranca@medicina.ufmg.br
}

\begin{abstract}
This population-based study aimed to determine the profile of early neonatal deaths in Belo Horizonte, Minas Gerais, Brazil, from 2000 to 2003. Profiles were analyzed from the perspective of avoidability, justified by persistently high early neonatal mortality rates in the city. Three profiles were generated for multiple causes of death from the perspective of fuzzy sets, using the Grade of Membership method. Birth weight and the hospital's corporate status were also related to the three profiles. Private hospitals were characterized by so-called "difficult-to-prevent deaths, with mention of congenital malformations" (profile 2). The Unified National Health System (SUS) generated two distinct profiles. Private maternity facilities contracted out by the SUS showed "preventable deaths" (profile 1), while "premature deaths" (profile 3) occurred in the public Federal and State maternity hospitals. This typology highlights the need to adopt differential policies in the SUS, focusing on evaluation and accreditation for maternity facilities contracted out by the SUS and-for the system as a whole - on the routine adoption of protocols for childbirth care and prophylactic measures that are known to reduce neonatal morbidity and mortality.
\end{abstract}

Infant Mortality; Early Neonatal Mortality; Multiple Causes of Death
Eliane de Freitas Drumond 1,2

Carla Jorge Machado 3

Elizabeth França ${ }^{2}$

\section{Introdução}

O Brasil é signatário dos Objetivos de Desenvolvimento do Milênio (ODM), compromisso proposto às nações pela Organização Mundial da Saúde no ano 2000 1. A redução da taxa de mortalidade infantil (TMI) até o ano 2015, uma das metas propostas pelos ODM, depende essencialmente da redução do componente neonatal precoce, atualmente responsável por mais da metade dos óbitos de crianças brasileiras no primeiro ano de vida 2 e estreitamente ligado a problemas na atenção à saúde da gestante e do recém-nascido ${ }^{3}$. No entanto, na complexa rede causal de determinação destes óbitos estão também envolvidos outros fatores, diretos e indiretos. Dentre esses, o peso de nascimento é a variável direta mais consistentemente associada a maior risco de morte neonatal e deve ser considerada nos estudos sobre mortalidade infantil 4 . A desigualdade sócio-econômica, principal fator determinante distal, tem sido relacionada a dificuldades de acesso e pior qualidade de atenção em saúde perinatal 5 . A adequação dos processos assistenciais pode ser avaliada por sua capacidade de interferir e reduzir os riscos de morte neonatal precoce, diretos ou indiretos. Esses processos assistenciais vão desde o atendimento individual até a organização, de forma hierarquizada e regionalizada, da rede de assistência à gestante, ao parto e ao recém-nascido. O elevado percentual de mortes nas primeiras horas de vida extra-ute- 
rina, em locais onde virtualmente todos os óbitos são hospitalares é, portanto, um indicador da importância de assistência hospitalar oportuna e adequada 6 ao binômio mãe-filho.

A abordagem segundo causas de morte é um dos mais importantes aspectos das análises de mortalidade. As causas básicas de morte utilizadas na divulgação das estatísticas de mortalidade são de fácil compreensão, mas podem representar significativa perda de informações, se comparadas à abordagem de causas múltiplas 7 . No caso específico dos óbitos neonatais precoces, esta abordagem pode ampliar a compreensão dos complexos patológicos que envolveram os binômios mãe-filho na gravidez e no parto. E também, ao possibilitar a avaliação da presença de exposição simultânea do recém-nascido a mais de um fator de risco, auxiliar as abordagens sob enfoque de evitabilidade ${ }^{8}$, especialmente aplicáveis no Brasil 9 .

A Conferência Internacional para a Décima Revisão da Classificação Internacional de Doenças 10, ao expressar seu estímulo à utilização da abordagem segundo causas múltiplas, não recomendou qualquer regra particular ou método de análise a serem seguidos. Os métodos devem se basear no pressuposto de que cada óbito é conseqüência de um conjunto de causas de morte, e não apenas de uma causa básica. A partir da teoria dos conjuntos nebulosos ("fuzzy sets") 11 foi desenvolvido o método Grade of Membership (GoM), que permite a análise de todas as causas de óbito mencionadas. Por esta lógica, pode-se assumir que uma população caracteriza-se por perfis extremos, representados por um grupo de causas de morte. Contudo, nem todos os indivíduos possuem exatamente as mesmas causas de morte do perfil extremo e isto é o mesmo que dizer que nem todos os indivíduos possuem inclusão - ou pertencimento - total a um dos perfis extremos característicos daquela população. Se um indivíduo tem pertencimento total a um perfil extremo, ele passa a ser considerado um tipo puro deste perfil. Para atender à diversidade de possibilidades de não pertencimento total a um perfil extremo assume-se, pelo método de GoM, que um indivíduo pode apresentar características de mais de um perfil e que é possível quantificar o seu grau de pertencimento a cada um dos perfis traçados para a população em estudo.

No Município de Belo Horizonte, Minas Gerais, Brasil, a redução da taxa de mortalidade neonatal precoce tem sido irregular e ela se mantém em níveis considerados ainda elevados (taxa de 7,4 por mil nascidos vivos em 2003) 12. A importância de se abordar este componente da TMI, onde há boa cobertura pré-natal e virtualmente todos os partos são hospitalares, foi evidenciada pelo elevado percentual de óbitos por causas consideradas evitáveis 13 , atribuídos especialmente a hipóxia ${ }^{9}$ no município. Neste sentido, o objetivo deste trabalho é descrever e analisar os perfis dos óbitos neonatais precoces ocorridos em Belo Horizonte, no período de 2000 a 2003, utilizando o enfoque de causas múltiplas de morte a partir da teoria dos conjuntos nebulosos, por faixa de peso e natureza jurídica do hospital.

\section{Material e métodos}

Analisaram-se inicialmente todos óbitos neonatais precoces de residentes em Belo Horizonte, ocorridos no período de 2000 a 2003, obtidos no Sistema de Informações sobre Mortalidade (SIM) da Secretaria de Saúde do município. O Sistema de Informação sobre Nascidos Vivos (SINASC) foi utilizado como fonte para resgate de pesos de nascimento em branco e para validação dos pesos registrados do SIM. Todos os óbitos excluídos tiveram peso de nascimento inferior a 500 gramas. A codificação das causas do óbito baseou-se na Classificação Internacional de Doenças - 10a Revisão (CID-10) 10. Foram consideradas causas múltiplas todas as causas mencionadas em cada uma das declarações de óbito, sem distinção da sua posição.

As variáveis utilizadas para determinação dos perfis de causas de morte foram as causas mencionadas nas declarações de óbito. Posteriormente, sobre os perfis determinados, foram relacionadas as freqüências de pesos de nascimento e as naturezas jurídicas dos estabelecimentos de ocorrência do óbito. Para análise da freqüência dos diagnósticos e da relação de suas menções como causa básica ou associada, todas as afecções mencionadas na declaração de óbito foram consideradas e arquivadas em campos distintos, de forma que cada linha reproduziu o conteúdo de uma declaração de óbito. Em seguida, foram excluídas as causas que, na mesma declaração de óbito, foram mencionadas mais de uma vez (duplicações) e as que eram semelhantes (multiplicações). Buscava-se, também, através desta avaliação inicial, que o maior conhecimento do padrão de causas de óbito da população possibilitasse a elaboração de uma lista reduzida dos grupos de causas, com as seguintes características: (1) ser definida a partir dos códigos da CID10; (2) refletir a freqüência e relevância dos diagnósticos mencionados nos atestados de óbito; (3) ter uma denominação a mais informativa possível sobre as diversas patologias e os códigos nela incluídos; (4) permitir a comparabilidade com outros dados; e (5) não conter número excessivo 
de categorias. A partir destas premissas e de adequação de proposta internacional para definição de grupos de causas ${ }^{14}$, foram obtidos seis grupos: imaturidade (códigos P00 a P15), hipóxia (P20 a $\mathrm{P} 21)$, outras respiratórias (P22 a P28), infecciosas (A50 a A64 e P35 a P39 e P77), malformações (Q00 a Q99) e demais causas (P29 e P50 a P96).

Para simplificar, os grupos de causas assim definidos e que compuseram a lista reduzida de causas serão chamados de causas de morte. Para evitar a multiplicação de diagnósticos causada pela agregação de causas, as duplicações foram eliminadas. Cada causa de morte recebeu, então, o código " 0 " ou " 1 " em cada uma das declarações de óbito. O código " 1 " indicou a menção de uma das causas de óbito daquele grupo de causas naquela declaração de óbito. Nos casos de declarações onde houve ausência de menção do grupo, o código foi igual a " 0 ".

Para o peso de nascimento foram definidas três categorias: menor que 1.500 gramas; de 1.500 gramas a 2.499 gramas e igual ou superior a 2.500 gramas.

Os hospitais de ocorrência dos óbitos foram categorizados, segundo sua natureza jurídica, como privados, públicos/universitários e contratados/conveniados ao Sistema Único de Saúde (SUS). Foram classificados na categoria privados, nove estabelecimentos de saúde que tinham como fontes de financiamento os pagamentos particulares por serviço e aqueles advindos dos chamados convênios de pré-pagamento. Entre os dezesseis hospitais categorizados como contratados/conveniados, estão os privados ou filantrópicos e que prestam serviço, sob forma de pós-pagamento, ao SUS. Na categoria públicos/ universitários, foram consideradas seis unidades (municipais, estaduais e federais) que compõem a rede própria do SUS de Belo Horizonte.

O método de GoM, um procedimento multivariado, foi utilizado para examinar perfis latentes de associação entre as causas de morte, para caracterizar em que medida os indivíduos se assemelham a estes perfis. Ele possibilita que as causas de morte de cada indivíduo sejam descritas de acordo com o grau de similaridade ou pertencimento aos aglomerados de causas comuns (ou típicos) encontrados 15. Os graus de similaridade entre aglomerados de causas e indivíduos somam a unidade para cada indivíduo e variam entre 0 e 1 , necessariamente 16 .

O ponto de partida do método é assumir a existência de K conjuntos nebulosos ("fuzzy sets") ou perfis extremos a serem definidos, tal que $\mathrm{k}=$ $1,2,3,4, \ldots, K$ 15,16. Cabe notar que estes conjuntos são chamados difusos por permitirem graus de pertencimento que podem ser parciais 11. A população estudada compreende I indivíduos
(I = 1, 2, 3,..., I), com J variáveis categóricas, onde a jésima variável possui lj níveis de resposta 15,16. Assume-se ainda que $\lambda_{\mathrm{kj} l}$ é a probabilidade de que a l-ésima resposta à variável j-ésima esteja associada com o k-ésimo conjunto nebuloso e que gik é o parâmetro que mensura o grau de similaridade das causas múltiplas de morte específicas de um dado indivíduo em relação às características de cada um dos K perfis extremos, com as seguintes restrições:

$$
\begin{aligned}
& 0 \leq \lambda_{k j l} \leq 1, \\
& \Sigma_{k} \lambda_{k j l}=1, \\
& 0 \leq g_{i k} \leq 1, \\
& \Sigma_{k} g_{i k}=1 .
\end{aligned}
$$

Assim, observa-se que o primeiro tipo de coeficientes, $\lambda_{\mathrm{kjl}}$ relaciona os $\mathrm{K}$ tipos puros às J variáveis observadas, enquanto que o segundo tipo de coeficientes, $g_{i k}$, descreve a distância ou similaridade entre as características observadas de um indivíduo em termos de causas múltiplas e as características de cada perfil extremo K 15,16.

$\mathrm{O}$ produto $\mathrm{g}_{\mathrm{ik}} \lambda_{\mathrm{kjl}}$ revela a probabilidade de que um indivíduo que pertence inteiramente ao perfil $\mathrm{k}$ (ou seja, apenas aqueles que se associam unicamente a um perfil extremo ou tipo puro) possua a resposta 1 para a questão j. A probabilidade que um indivíduo tenha uma resposta 1 à questão $\mathrm{j}, \mathrm{p}\left(\mathrm{x}_{\mathrm{ijl}}\right)=1$, é a soma deste produto para todos os perfis de GoM gerados (K perfis) 16,17. Esta probabilidade é dada por

$$
P\left(x_{i j l}=1,0\right)=\Sigma_{k} g_{i k} \lambda_{k j l} \text {. }
$$

E, finalmente, assumindo independência das observações individuais, condicionalmente a $\lambda_{\mathrm{kjl}}$ e $g_{\mathrm{ik}}$, a função de verossimilhança fica assim definida:

$$
L=\Pi_{i} \Pi_{j} \Pi_{l}\left(\Sigma_{k} g_{i k} \lambda_{k j l}\right) x_{i j l}
$$

Essa função fica sujeita às seguintes restrições:

$0 \leq \lambda_{k j l} \leq 1$,

$\Sigma_{k} \lambda_{k j l}=1$,

$0 \leq g_{i k} \leq 1$,

$\Sigma_{k} g_{i k}=1$.

Os parâmetros g $g_{\mathrm{ik}}$ e $\lambda_{\mathrm{kjl}}$ foram estimados, de forma iterativa, utilizando o programa GOM3. EXE (http://lib.stat.cmu.edu/DOS/general/.index.html), executável em ambiente DOS. O programa encontra-se disponível na internet. Estimou-se o modelo em dois níveis ${ }^{17}$. No primeiro nível, estimaram-se os parâmetros $\lambda_{\mathrm{kjl}}$ e $\mathrm{g}_{\mathrm{ik}}$ a partir das causas de morte (variáveis internas), ou seja, excluindo as variáveis natureza do estabelecimento e peso (variáveis externas). Em seguida, a matriz de $g_{i k}$ foi fixada e um novo modelo ajustado, com novos valores de $\lambda_{\mathrm{kjl}}$ obtidos para as variáveis natureza do estabelecimento e peso ao nascer (variáveis externas ao modelo). Assim, as causas de morte fazem parte da conformação dos perfis e dos graus de pertencimento, en- 
quanto que as estimativas de $\lambda_{\mathrm{kjl}}$ obtidas para as variáveis remanescentes foram empregadas como uma aproximação de variáveis de estratificação de cada um dos perfis extremos 15,16. A idéia é que se deseja conhecer as probabilidades de que cada um dos indivíduos tipos puros de um perfil extremo K tenha um nível l de uma variável externa J. Cabe comentar que as categorias de peso ao nascer e de natureza do estabelecimento foram definidas como variáveis externas porque o objetivo do estudo é entender como as causas múltiplas aparecem de forma conjunta. Assim, desejava-se encontrar "fuzzy sets" para os quais os graus de pertencimento pudessem ser especificados apenas em termos das variáveis internas (as condições mórbidas no momento da morte). As variáveis externas são aquelas que, ainda que relacionadas aos conjuntos formados, são de outra natureza e não entram na conformação dos graus de pertencimento. A utilização de uma ou mais variáveis externas se justifica como forma de validação dos perfis encontrados 18. Quanto à variável sexo, deve ser ressaltado ainda que, nesse estudo, a não utilização desta variável foi uma decisão tomada, também, com base nos seguintes aspectos: não ter havido diferença na taxa de mortalidade entre os sexos, por faixa de peso, conforme tabulações preliminares dos dados realizadas pelos autores; pelo fato de os critérios de evitabilidade comumente adotados não considerarem essa variável 19 e, tendo em vista que neste estudo se propôs, principalmente, a discussão de variáveis assistenciais envolvidas na determinação dos óbitos. Assim, a abordagem dos mecanismos biológicos ultrapassaria seu escopo.

O número ótimo de perfis pode ser determinado tanto por quão interpretável é um determinado conjunto de K perfis, do ponto de vista teórico e conceitual, quanto por um critério estatístico - o teste da razão de verossimilhança, que mede a variação no poder explicativo entre os modelos com $\mathrm{K}$ e $\mathrm{K}+1$ perfis extremos. Esta razão segue uma distribuição qui-quadrado, com número de graus de liberdade igual à diferença no número de parâmetros a serem estimados entre os modelos 16:

$$
\mathrm{df}=(\mathrm{I}-1)+\Sigma_{j} L_{j}
$$

A hipótese nula, ao proceder ao teste da razão de verossimilhança, é que o modelo com $\mathrm{K}+1$ perfis não adiciona poder explicativo a um modelo com K perfis.

\section{Resultados}

Do total de 998 óbitos neonatais precoces ocorridos no período, foram selecionados 875 para inclusão no estudo. Nos hospitais da rede privada ocorreram $11 \%(n=92)$, na rede contratada/conveniada $43 \%(n=379)$ e nos hospitais da categoria público/universitário 46\% (n = 404) dos óbitos. Em relação ao sexo e ao peso, 53,3\% ( $\mathrm{n}=467$ ) dos óbitos foram de recém-nascidos do sexo masculino e $59,2 \%(n=518)$ apresentaram peso ao nascer inferior a 1.500g. Encontraram-se 2.481 menções de causas de morte, com variação entre uma e seis causas de morte por declaração e média de 2,8 .

De acordo com o método de GoM e os critérios de perfis interpretáveis e estatísticos, concluiu-se que três perfis representavam adequadamente o panorama de co-morbidade, a partir das causas de morte mencionadas. A Tabela 1 mostra as relações entre os perfis extremos e as variáveis indicadoras de presença das causas de morte através das probabilidades $\lambda_{\mathrm{kj}}$. Nas primeiras colunas estão o número e a proporção de recém nascidos que faleceram com e sem menção de cada uma das causas. As demais colunas são as associadas com cada perfil extremo especificamente e revelam $\lambda_{\mathrm{kjl}}$, a probabilidade de que a presença ou ausência da causa de morte esteja associada com um determinado perfil extremo. Os perfis podem ser descritos através da comparação da diferença em valores entre as freqüências relativas da população de recém nascidos em estudo na primeira coluna com os $\lambda_{\mathrm{kj} l}$ nas demais colunas. As últimas duas variáveis apresentadas são as variáveis externas ao modelo, com suas respectivas probabilidades de que a presença ou ausência da causa múltipla esteja associada com um determinado perfil extremo, ou seja, $\lambda_{\mathrm{kj}}$.

Em relação aos perfis, observa-se que os recém nascidos tipos puros do perfil extremo 1 ou, em outras palavras, com grau de pertencimento total ao Perfil 1 não possuíam menção de prematuridade e nem de malformação congênita. Tenderam a ter mais menções de hipóxia, de outras respiratórias, de doenças infecciosas e de demais afecções, tanto em relação a qualquer recém-nascido tomado ao acaso desta população quanto em relação aos tipos puros de qualquer dos outros dois perfis. Denominou-se este perfil de "óbitos passíveis de prevenção".

Os recém-nascidos com grau de pertencimento total ao Perfil 2 não possuíam menção de hipóxia. Todos tiveram menção de malformação congênita. Tenderam a ter um menor número de menções de outras afecções respiratórias e infecciosas, relativamente à população como um todo e aos tipos puros dos demais perfis extremos. Devido à presença de malformação congênita em todos os tipos puros deste perfil, este foi denominado de "óbitos dificilmente preveníveis, com menção de malformação”. 
Coeficientes ( $\lambda \mathrm{kjl}$ ) das variáveis internas e externas para cada perfil de óbito neonatal precoce. Belo Horizonte, Minas Gerais, Brasil, $2000-2003$.

\begin{tabular}{|c|c|c|c|c|c|}
\hline Variável & Freqüência absoluta & Freqüência relativa & $\begin{array}{c}\text { Perfil } 1 \\
\mathrm{I}_{1 \mathrm{jl}}\end{array}$ & $\begin{array}{c}\text { Perfil } 2 \\
\mathrm{I}_{2 j l}\end{array}$ & $\begin{array}{c}\text { Perfil } 3 \\
\mathrm{I}_{3 \mathrm{jl}}\end{array}$ \\
\hline \multicolumn{6}{|l|}{ Grupos de causas } \\
\hline \multicolumn{6}{|l|}{ Prematuridade } \\
\hline Sim & 620 & 0,71 & 0,00 & 0,48 & 1,00 \\
\hline Não & 255 & 0,29 & 1,00 & 0,52 & 0,00 \\
\hline \multicolumn{6}{|l|}{ Hipóxia } \\
\hline Sim & 132 & 0,15 & 0,54 & 0,00 & 0,50 \\
\hline Não & 743 & 0,85 & 0,46 & 0,63 & 0,46 \\
\hline \multicolumn{6}{|l|}{ Outras respiratórias } \\
\hline Sim & 539 & 0,60 & 0,52 & 0,37 & 0,54 \\
\hline Não & 336 & 0,40 & 0,48 & 0,63 & 0,46 \\
\hline \multicolumn{6}{|l|}{ Infecciosas } \\
\hline Sim & 210 & 0,24 & 0,49 & 0,32 & 0,43 \\
\hline Não & 665 & 0,76 & 0,51 & 0,68 & 0,57 \\
\hline \multicolumn{6}{|l|}{ Malformações } \\
\hline Sim & 171 & 0,19 & 0,00 & 1,00 & 0,00 \\
\hline Não & 704 & 0,81 & 1,00 & 0,00 & 1,00 \\
\hline \multicolumn{6}{|l|}{ Demais } \\
\hline Sim & 310 & 0,35 & 0,51 & 0,49 & 0,43 \\
\hline Não & 56.5 & 0,65 & 0,49 & 0,51 & 0,57 \\
\hline \multicolumn{6}{|l|}{ Variáveis externas } \\
\hline \multicolumn{6}{|l|}{ Natureza jurídica } \\
\hline Privado & 96 & 0,11 & 0,00 & 0,32 & 0,07 \\
\hline Contratado/Convênio & 376 & 0,43 & 1,00 & 0,00 & 0,37 \\
\hline Público & 403 & 0,46 & 0,00 & 0,68 & 0,56 \\
\hline \multicolumn{6}{|l|}{ Peso (gramas) } \\
\hline$\leq 1.500$ & 516 & 0,59 & 0,00 & 0,00 & 1,00 \\
\hline $1.500-2.499$ & 175 & 0,20 & 0,00 & 1,00 & 0,00 \\
\hline$\geq 2.500$ & 184 & 0,21 & 1,00 & 0,00 & 0,00 \\
\hline
\end{tabular}

Fonte: Sistema de Informações sobre Mortalidade (Secretaria Municipal de Saúde de Belo Horizonte).

Finalmente, os recém nascidos com grau de pertencimento total ao Perfil 3 eram invariavelmente prematuros. Não possuíam menção de malformação congênita. Tenderam a ser hipóxicos e a ter outras afecções respiratórias. Este perfil foi denominado de "óbitos de prematuros".

Observa-se, a seguir, que todos os "óbitos passíveis de prevenção”, dos tipos puros do Perfil 1 , tinham peso de nascimento acima de $2.500 \mathrm{~g}$ e ocorreram em hospitais contratados/conveniados. Já os tipos puros do Perfil 2 tenderam a ser óbitos ocorridos em hospitais privados ou em hospitais públicos, em detrimento de hospitais contratados/conveniados. Assim, interpreta-se que na população em estudo $11 \%$ dos óbitos ocorreram em hospitais privados, $43 \%$ em hospitais contratados/conveniados e $46 \%$ em hospitais públicos, mas se um recém-nasci- do é um tipo puro do Perfil 2, o óbito tem $32 \%$ de probabilidade de ter ocorrido em hospital privado e $68 \%$ de probabilidade de ter ocorrido em hospital público, sendo nula a probabilidade de ter ocorrido em hospital contratado/conveniado. Todos os tipos puros deste perfil extremo nasceram com peso entre $1.500 \mathrm{~g}$ e $2.499 \mathrm{~g}$. Finalmente, os tipos puros do Perfil 3 tiveram maior probabilidade de ocorrer em hospitais públicos, em detrimento tanto dos privados quanto dos contratados/conveniados. Todos os óbitos foram de recém-nascidos cujo peso ao nascimento foi inferior a $1.500 \mathrm{~g}$.

A Tabela 2 mostra a distribuição dos escores $\mathrm{g}_{\mathrm{ik}}$ na população estudada, para cada perfil. Observa-se que $48,4 \%(n=424)$ dos óbitos infantis teve pertencimento total ao Perfil 3, o que implica nas seguintes probabilidades, associadas 
Tabela 2

Distribuição dos óbitos segundo intervalos de escores $\left(g_{i k}\right)$ para cada perfil de óbito neonatal precoce. Belo Horizonte, Minas Gerais, Brasil, 2000-2003.

\begin{tabular}{lccc}
\hline Intervalos de $\mathbf{g}_{\mathrm{ik}}{ }^{*}$ & Perfil 1 & Perfil 2 & Perfil 3 \\
\hline 0,00 & 81,3 & 61,7 & 32,8 \\
$0,01-0,24$ & 0,1 & 18,9 & 2,6 \\
$0,25-0,49$ & 0,0 & 2,6 & 0,0 \\
$0,50-0,74$ & 1,7 & 0,0 & 0,9 \\
$0,75-0,99$ & 3,5 & 2,7 & 15,3 \\
1,00 & 13,4 & 14,1 & 48,4 \\
Total & 100,0 & 100,0 & 100,0 \\
Prevalência (\%) ** & 17,4 & 18,9 & 63,7 \\
\hline
\end{tabular}

* Intervalos de graus de pertencimento dos óbitos a cada um dos perfis;

** Prevalência ponderada pelo grau de pertencimento dos indivíduos ao perfil (\%).

Fonte: Sistema de Informações sobre Mortalidade (Secretaria Municipal de Saúde de Belo Horizonte) dos perfis. Pode-se dizer que esses escores médios corresponderam, então, à prevalência ponderada de cada um dos perfis na população e que, desta forma, o Perfil 3 é o de maior prevalência, seguido pelo Perfil 2 e pelo Perfil 1 (Tabela 2).

\section{Discussão}

Análises de causas básicas de óbitos neonatais precoces, comumente classificadas segundo grandes agrupamentos como as afecções perinatais e as malformações congênitas, podem ser pouco elucidativas por não se relacionarem à possibilidade de sua prevenção 5,10. A utilização de todas as causas mencionadas nas declarações de óbito minimiza dificuldades, muitas vezes relatadas pelos médicos, na identificação da melhor seqüência que descreveria a sucessão de eventos que culminou na morte. Essas dificuldades para a ordenação das causas têm reflexo sobre as regras de seleção de causas básicas, internacionalmente adotadas, que levam em conta a posição na qual as afecções foram citadas. Por isto, é possível que não sejam selecionadas como causas básicas, após aplicação das regras de seleção, aquelas causas que seriam eleitas pelo médico declarante.

Para Santo \& Pinheiro 20, a análise do número médio de diagnósticos mencionados na declaração de óbito é de fundamental importância já que, além de outros aspectos, poderá revelar tanto a qualidade da assistência médica recebida como a qualidade do preenchimento da declaração. Neste estudo, após a exclusão das duplicidades e multiplicações de causas, verificou-se uma média de 2,8 diagnósticos por declaração de óbito, que é comparável às médias obtidas para as doenças crônico-degenerativas 21, para as quais a abordagem de causas múltiplas tem sido mais freqüentemente adotada. Por outro lado, em estudo de confiabilidade da causa básica de óbito realizado em 13 cidades da região metropolitana de Belo Horizonte, os autores observaram que havia subestimação do agrupamento relativo às afecções maternas 22 . Nesse agrupamento se incluem causas de morte que são passíveis de prevenção por adequada atenção pré-natal. Assim, sob o enfoque da evitabilidade, os resultados atualmente obtidos poderiam estar subestimando este agrupamento de causas. Portanto, a qualificação dos dados de mortalidade e em especial das causas de morte, uma das ações prioritárias dos gestores dos sistemas de informação em saúde, é ferramenta básica para análises da situação de saúde de uma população.

A utilização da teoria dos conjuntos nebulosos ("fuzzy sets"), através da aplicação da meto- 
dologia do GoM sobre os grupos de causas de óbitos, com a posterior agregação do peso de nascimento e da natureza jurídica dos hospitais de ocorrência a esses perfis, possibilitou a maximização da utilização das informações contidas nas declarações de óbito. Assim, delinearam-se três distintos perfis de causas, com grandes diferenças nos padrões de freqüência de patologias que levaram à morte e aos quais os recém-nascidos podiam ter diferentes graus de pertencimento. A incorporação das informações relativas às variáveis externas "peso de nascimento" e "natureza jurídica do estabelecimento de saúde", tornou ainda mais nítidas as diferenças entre os três perfis e também foi útil no sentido de validar os perfis de causas encontrados. A título de exemplo, observa-se que um dos perfis encontrados foi definido como sendo de prematuros (todos os óbitos com pertencimento total a este perfil eram de prematuros). Dado que os prematuros tendem a ter baixo peso, o fato de tipos puros deste perfil terem sido todos de menos de $1.500 \mathrm{~g}$ ao nascimento é uma evidência de que o perfil encontrado é condizente com o esperado.

Para análises de causas múltiplas há principalmente duas propostas 20,23. A primeira referese às análises da freqüência de cada diagnóstico como causa básica ou associada, mais facilmente acessíveis em termos de saúde pública. A outra proposta é ainda de uso restrito: são os estudos de associações de causas nos quais se parte da premissa de interação entre as causas. Ainda não há conceituação e procedimentos para codificação e tabulação internacionalmente padronizadas e os métodos de análise baseados na independência das variáveis não se aplicam às análises de associações de causas. A opção aqui adotada, o método de GoM, permitiu a sistematização das causas múltiplas de morte em conjuntos de enfermidades que se associavam entre si, desdobrando-se em manifestações de doenças que conduziram à morte. Observou-se com mais clareza e simplicidade os conjuntos de enfermidades que levaram ao óbito. Para abordagens em Saúde Pública é mais elucidativo entender como a confluência de doenças compõe mecanismos de causação de mortes e, uma vez conhecidas estas etapas, tornam-se mais evidentes as medidas a serem tomadas para evitar o óbito. Esta abordagem vai além de análises conhecidas. Podem-se citar análises baseadas no cálculo do "Q de Yule" para verificação da magnitude da associação de causas (básicas e associadas) 24,25, que parte da pressuposição de ocorrência independente das doenças, o que claramente não é verdadeiro no caso das causas múltiplas para um indivíduo.

Através do método de GoM também foi possível definir para cada óbito seu grau de perten- cimento a cada um dos conjuntos formados. Isso indicou a menor ou maior prevalência dos perfis na população. É importante frisar que a obtenção de parâmetro individual como este implica no reconhecimento das diferenças entre indivíduos e dos diferentes caminhos e associações de mesma causa de morte. Ou seja, admite-se que há uma heterogeneidade mensurável na população em estudo.

É importante ressaltar também que são encaminhadas gestantes, parturientes e recém-nascidos de maior risco para os ambulatórios e hospitais públicos da rede SUS de Belo Horizonte. Esses hospitais são também referências para tratamentos de infertilidade e patologias fetais. Já os partos de risco habitual são mais freqüentemente realizados na rede contratada/conveniada. Chamam atenção, especialmente, as características dos recém-nascidos pertencentes ao Perfil 1 ( $\mathrm{n}=164)$, de "óbitos passiveis de prevenção": pesos de nascimento maiores que $2.500 \mathrm{~g}$, elevada probabilidade de ocorrência de hipóxia, todos ocorridos em hospitais contratados/conveniados. As freqüentes associações de menções de infecção e demais causas não foram suficientes para elucidar a etiologia dos quadros de hipóxia, e não contribuíram para melhor localização das possibilidades de falhas, se na integração da rede de assistência à gestante e ao recém-nascido ou na identificação e minimização de riscos de morte. Vale ressaltar que óbitos em maiores de $2.500 \mathrm{~g}$ são considerados eventos-sentinela em saúde, relacionados a prováveis falhas no manejo obstétrico ou na assistência neonatal ${ }^{19}$. Problemas no atendimento à parturiente podem ser devidos a dificuldades de acesso tempestivo à assistência obstétrica, em conseqüência da falta de percepção da gravidade do caso pela parturiente e sua família ou pela já conhecida peregrinação em busca de assistência obstétrica qualificada a que as parturientes são submetidas 26. Podem, também, ser decorrentes da subestimação de riscos já detectados no pré-natal ou da inexistência/inadequação de protocolos hospitalares para avaliações durante o trabalho de parto, capazes de alertar quando há aumento do risco no préparto.

Por outro lado, em cerca da metade das mortes incluídas no Perfil 3, "óbitos de prematuros", houve associação de causas típica de países onde a mortalidade é elevada: hipóxia, outras doenças respiratórias e infecção, principalmente. Esses óbitos ocorreram na rede SUS, com maior proporção em hospitais públicos. A despeito da observada tendência de aumento de nascimentos pré-termo no mundo e no Brasil 27,28, as dificuldades inerentes à correta determinação da idade gestacional podem ter levado à inclusão neste 
grupo de crianças a termo, com retardo de crescimento intra-uterino. Um acompanhamento pré-natal adequado pode diminuir a incidência de baixo peso ao nascer, reduzindo tanto os casos de retardo do crescimento intra-uterino quanto os de prematuridade evitável. A associação de menções de doenças respiratórias e infecciosas com o perfil "óbitos de prematuros" faz supor que, mesmo com a comprovação dos efeitos da corticoterapia antenatal 29 e da antibioticoterapia profilática intraparto ${ }^{30}$ na redução da morbimortalidade neonatal, a cobertura nessa população seja baixa. Finalmente, a impossibilidade de disponibilização de suporte avançado de vida para estas crianças poderia estar ligada às características da estruturação das unidades de terapia intensiva neonatal relacionadas por Barbosa 31: falta de eqüidade na distribuição dos leitos, limitação do acesso para parcela mais carente da população e qualidade irregular dos serviços prestados.

Apesar de a classificação dos hospitais aqui utilizada poder representar uma limitação para essa análise, pois poderiam estar incluídos serviços com características diferentes em uma mesma categoria, a observação desta tipologia na rede SUS de Belo Horizonte aponta para a necessidade de abordagens específicas para pre- venção dos óbitos neonatais precoces, segundo sua natureza jurídica. Nos hospitais públicos, referências para partos de risco, deve ser incentivado o uso rotineiro de medidas profiláticas de reconhecido impacto sobre a mortalidade de prematuros. A associação entre óbitos de crianças de baixo risco aos hospitais contratados/ conveniados ao SUS aponta para a necessidade de reavaliação destas instituições, visando especialmente à adequação da utilização de tecnologias, incluídos aqui tanto as estruturas hospitalares quanto os protocolos de atendimento ao parto e ao recém-nascido.

Para que o efeito protetor da ampla cobertura da assistência pré-natal em Belo Horizonte se estenda a todo ciclo gravídico-puerperal, as intervenções oportunas e qualificadas no trabalho de parto e na assistência imediata ao recémnascido também devem estar disponíveis para todos. A ocorrência da quase totalidade dos partos em ambiente hospitalar no período estudado é evidência da suficiência do número de leitos e do desejo da população de buscar os hospitais como locais com potencial para reduzir os riscos de morbi-mortalidade do binômio mãe-filho. É eticamente inaceitável, portanto, a ocorrência de óbitos potencialmente evitáveis na proporção detectada neste estudo.

\section{Resumo}

Estudo de base populacional para determinar perfis de óbitos neonatais precoces ocorridos em Belo Horizonte, Minas Gerais, Brasil, de 2000 a 2003. A definição dos perfis de causas amplia possibilidade de análise sob enfoque de evitabilidade, justificada pela persistência de altas taxas de mortalidade neonatal precoce. Três perfis foram gerados, a partir do enfoque de causas múltiplas, sob a perspectiva dos conjuntos nebulosos ("fuzzy sets"), utilizando-se técnica Grade of Membership. Relacionaram-se esses perfis ao peso de nascimento e a natureza jurídica do hospital de ocorrência do óbito. Nos hospitais da rede privada ocorreram "óbitos dificilmente preveníveis, com menção de malformação congênita" (perfil 2). Aos hospi- tais do Sistema Único de Saúde (SUS) associaram-se dois perfis distintos. Nos hospitais contratados/conveniados ocorreram os "óbitos passíveis de prevenção" (perfil 1) e na rede própria os "óbitos de prematuros" (perfil 3). A tipologia observada aponta para necessidade de adoção de políticas diferenciadas na rede SUS: prioritariamente, na rede contratada/conveniada, ações voltadas para credenciamento e avaliação da qualidade da assistência; e, em toda rede, adoção rotineira de protocolos assistenciais e medidas profiláticas, redutores da morbimortalidade neonatal.

Mortalidade Infantil; Mortalidade Neonatal Precoce; Causas Múltiplas de Morte 


\section{Colaboradores}

E. F. Drumond participou de todas as etapas do estudo e da elaboração do artigo, sendo responsável pela versão final. C. J. Machado contribuiu na análise, discussão dos resultados e revisão do manuscrito. E. França contribuiu na discussão dos resultados e revisão crítica do manuscrito. Todas as autoras participaram da concepção do trabalho.

\section{Referências}

1. World Health Organization. Objectifs du Millénaire pour ler développement. http://www.un.org/ french/millenniumgoals/index.html (acessado em 02/Abr/2005).

2. Ministério da Saúde. Saúde Brasil 2004: uma análise da situação de saúde. Brasília: Ministério da Saúde; 2004.

3. Tinker A, ten Hoope-Bender P, Azfar S, Bustreo F, Bell R. A continuum of care to save newborn lives. Lancet 2005; 365:822-5.

4. Wilcox AJ. On the importance - and the unimportance - of the birthweight. Int J Epidemiol 2001; 30:1233-41.

5. Lawn JE, Cousens S, Zupan J. 4 million neonatal deaths: When? Where? Why? Lancet 2005; 365: 891-900.

6. Leal MC, Gama SGN, Campos MR, Cavalini LT, Garbayo LS, Brasil CLP, et al. Fatores associados à morbi-mortalidade perinatal em amostra de maternidades públicas e privadas do Município do Rio de Janeiro, 1999-2001. Cad Saúde Pública 2004; 20 Suppl 1:20-33.

7. Niobey FML, Cascão AM, Duchiade ML, Sabroza PC. Qualidade do preenchimento de atestados de óbitos de menores de um ano na região metropolitana do Rio de Janeiro. Rev Saúde Pública 1990; 24:311-8.

8. Machado CJ. Mortalidade infantil no Estado de São Paulo, 1999: uma análise das causas múltiplas de morte a partir dos componentes principais. Rev Bras Epidemiol 2004; 7:11-21.

9. Lansky S, França E, Leal MC. Mortes perinatais evitáveis em Belo Horizonte, Minas Gerais, Brasil, 1999. Cad Saúde Pública 2002; 18:1389-400.

10. Organização Mundial da Saúde. Classificação Estatística Internacional de Doenças e Problemas Relacionados à Saúde - 10o revisão. v. 1. São Paulo: Edusp; 2000.

11. Zadeh LA. Probability measures and fuzzy events. J Math Anal Appl 1968; 23:421-7.

\section{Agradecimentos}

Este trabalho contou com financiamento do Conselho Nacional de Desenvolvimento Científico e Tecnológico (processo n. 403707/04-8).
12. Secretaria Municipal de Saúde de Belo Horizonte. Boletim epidemiológico: mortalidade infantil em Belo Horizonte. Belo Horizonte: Secretaria Municipal de Saúde de Belo Horizonte; 2004.

13. Caldeira AP, França E, Perpétuo IH, Goulart EMA. Evolução da mortalidade infantil por causas evitáveis, Belo Horizonte, 1984-1998. Rev Saúde Pública 2005; 39:67-74.

14. Centers for Disease Control and Prevention/National Center for Health Statistics. Proceedings of the International Collaborative Effort on Perinatal and Infant Mortality. http://www.cdc.gov/ nchs/data/misc/ice92_3acc.pdf (acessado em 19/ Ago/2006).

15. Manton KG, Woodbury MA, Tolley HD. Statistical applications using fuzzy sets. New York: John Wiley \& Sons; 1994.

16. McNamee P. A comparison of the grade of membership measure with alternative health indicators in explaining costs for older people. Health Economics 2004; 13:379-95.

17. Sawyer DO, Leite IC, Alexandrino R. Perfis de utilização de serviços de saúde no Brasil. Ciênc Saúde Coletiva 2002; 7:757-76.

18. Cassidy F, Pieper CF, Carroll BJ. Subtypes of mania determined by grade of membership analysis. Neuropsychopharmacology 2001; 25:373-83.

19. Wigglesworth JS. Monitoring perinatal mortality: a pathophysiological approach. Lancet 1980; 2:684-6.

20. Santo AH, Pinheiro CE. Multiple causes-of-death tabulator. Rev Bras Epidemiol 1999; 2:90-7.

21. Rezende EM, Sampaio IBM, Ishitani LH. Causas múltiplas de morte por doenças crônico-degenerativas: uma análise multidimensional. Cad Saúde Pública 2004; 20:1223-31.

22. França E, Goulart EMA, Machado JAD.Confiabilidade da declaração de causa básica de mortes infantis em região metropolitana do sudeste do Brasil. Rev Saúde Pública 1994; 28:385-91. 
23. Laurenti R, Buchalla CM. Elaboração de estatísticas de mortalidade segundo causas múltiplas. Rev Bras Epidemiol 2000; 3:21-8.

24. Saad PM. Mortalidade infantil por causas no Estado de São Paulo (Brasil) em 1983: análise sob a perspectiva das causas múltiplas de morte. Rev Saúde Pública 1986; 20:481-8.

25. Niobey CS. A análise da mortalidade infantil pósneonatal sob a perspectiva das causas múltiplas [Dissertação de Mestrado]. Rio de Janeiro: Escola Nacional de Saúde Pública, Fundação Oswaldo Cruz; 1994.

26. Tanaka ACA. Maternidade dilema entre nascimento e morte. São Paulo/Rio de Janeiro: Editora Hucitec; 1995.

27. Barbieri MA, Silva AAM, Bettiol H, Gomes UA. Fatores de risco para a tendência ascendente do baixo peso ao nascer em nascidos vivos de parto vaginal no Sudeste do Brasil. Rev Saúde Pública 2000; 34:596-602.
28. Barros F, Diaz-Rossello JL. Redes multicêntricas e qualidade da atenção neonatal. J Pediatr (Rio J) $2004 ; 80: 254-5$

29. Hallliday HL. Use of steroids in the perinatal pe riod. Paediatr Respir Rev 2004; 5 Suppl A:S321-7.

30. Lukacs SL, Schoendorf KC, Schuchat A. Trends in sepsis-related neonatal mortality in the United States, 1985-1998. Pediatr Infect Dis J 2004; 23:599603.

31. Barbosa AP. Terapia intensiva neonatal e pediátrica no Brasil: o ideal, o real e o possível. J Pediatr (Rio J) $2004 ; 80: 437-8$.

Recebido em 06/Mai/2005

Versão final reapresentada em 16/Jan/2006

Aprovado em 20/Abr/2006 